\title{
CONVERSIÓN AUTOMÁTICA DE FECHAS ISLÁMICAS A CRISTIANAS
}

Por

MARIA LUISA ÁVILA

En las páginas que siguen presento el programa para ordenador "Conversor" elaborado por la autora de este artículo y por Luis Molina. Pero antes de entrar en la descripción de dicho programa quisiera hacer algunas reflexiones sobre el papel que puede y debe jugar la informática en la investigación en el ámbito de las Humanidades.

\section{El ordenador personal y la investigación}

La utilización del ordenador en todos los campos de la investigación es un hecho desde hace muchos años, incluso en Humanidades (1), por lo que sería ocioso hablar ahora sobre esa cuestión. Pero en los últimos años - en el caso de España podríamos referirnos a los dos o tres últimos años - se ha producido una revolución, más sociológica que tecnológica, en la incidencia de la informática en la vida diaria: la aparición en el mercado de microordenadores de muy reducido coste y de prestaciones nada despreciables. En nuestro país dos han sido los modelos que han conocido una mayor difusión: el Commodore 64 y el Spectrum de Sinclair, que es el que hemos utilizado en esta ocasión.

Este hecho obliga a revisar a fondo las relaciones entre la investigación en Humanidades y la informática. Hasta ahora sólo un grupo muy reducido de historiadores, filólogos, etc. se habian atrevido a acercarse a un ordenador. Los grandes centros de cálculo eran vistos como algo distante $y$ en ocasiones, de dificil acceso. El investigador, casi siempre desconocedor de las técnicas informáticas, debía ponerse totalmente en manos del programador correspondiente $y$ ajustar su ritmo de trabajo a las necesidades del centro de cálculo. Es indudable que para el tratamiento de grandes cantidades de datos sigue siendo necesario recurrir a entidades que dispongan de ordenadores de tipo medio $y$ alto, pero alguno de los trabajos que antes se encomendaban a dichos ordenadores $y$, sobre todo, multitud de tareas que parecían dema-

11) En el campo del arabisamo habria que resaltar el proyecto internacional Onomasticon Arabicum, coordinado por el CNRS, en el que participa un equipo español dirigido por la doctora Marín Niño. 
siado insignificantes para tomarse las molestias que suponía acceder a un centro de cálculo, pueden ser perfectamente realizadas con la nueva generación de ordenadores personales y microordenadores. Un ejemplo de ello es el programa que aquí presento, pero quisiera apuntar un par de ideas sobre aplicaciones del ordenador personal a nuestro campo de investigación: los ficheros bibliográficos y los tratamientos de textos.

Desde hace algunos años, el instituto de Formación y Documentación en Ciencias Sociales y Humanidades (ISOC) del CSIC viene publicando el Indice Español de Humanidades (2). Con todos los artículos de una larguísima serie de revistas españolas se confecciona un índice de materias formado por palabras clave que describen con bastante exactitud el contenido del artículo. Esta magnífica labor del ISOC se va convirtiendo con el paso del tiempo en una ayuda insustituible para el investigador, pero cuando se alcanza un alto grado de especialización en un campo determinado y muy concreto, las palabras clave del IEH resultan demasiado generales. Para un estudioso de la historiografía hispanoárabe del siglo XI no es muy útil hallar un artículo sobre este tema descrito con las palabras clave Al-Andalus/Historiografía/Fuentes/siglo XI. A pesar de ello, y como muestra de la innegable calidad del trabajo, incluso en estos casos la utilidad del IEH sigue siendo apreciable. Sin embargo, za quién no le gustaría contar con un fichero bibliográfico especializado que, siguiendo el método de palabras clave y descriptores, le permitiese manejar con suma facilidad unos cuantos centenares de títulos? Añádase a esto la comodidad de poder elaborar índices bibliográficos, seleccionando de nuestro fichero los títulos que han de ser incluídos, sin tener que escribir una sola línea, ya que la impresora se encarga de librarnos de esa tarea.

Visto desde la perspectiva del ahorro del trabajo mecánico y rutinario, más interesante aún puede ser un buen programa de tratamiento de textos. En primer lugar, el texto en cuestión sólo deberá ser escrito una vez, pudiendo posteriormente ser ampliado, corregido o modificado cuantas veces sea necesario. Pero esto mismo se puede conseguir incluso con algunas máquinas de escribir. Lo que convierte un programa de tratamiento de textos en algo realmente útil es su capacidad para "leer» el texto almacenado en la memoria del ordenador en breves instantes. De esta forma el índice más completo que imaginarse pueda queda superado por un programa de este tipo. En los índices de cualquier obra, aparte de los nombres de persona, lugar, colectividades, títulos, etc. es frecuente que aparezca uno de términos técnicos, que nos permite localizar en el texto una serie de palabras que quien ha elaborado los indices estima de interés. Pero ocurre a veces que el criterio de esa persona no coincide con el de quien acude a esa obra en busca de datos sobre cierto término, encontrándose con que éste no se halla en los índices. Si el texto se conserva en soporte informático, quien accede a él tiene la posibilidad de localizar en unos instantes cualquiera de las palabras que forman dicho texto. Pero estos programas no sólo superan a los indices convencionales, sino que también pueden elaborarlos automáticamente. Me extendería demasiado si pasara a explicar el proceso a seguir; lo que ahora nos interesa es saber que un buen tratamiento de textos puede confeccionar con rapidez, seguridad y claridad cualquier tipo de índices (3).

Pero los filólogos - y en concreto los arabistas - nos hallamos siempre ante un serio problema a la hora de utilizar estas máquinas: la inclusión de los signos de trans-

12) En el momento de redactar estas líneas han aparecido los volúmenes correspondientes a la bibliografía de los años 1977 a 1980 .

(3) Quizá la mayor utilidad de la informática para confeccionar índices se manifieste en los índices KWIC y KWOC que utilizan los grandes abstracs y repettorios y que sería interesante que se extendieran a otro tipo de obras. 
cripción. Pues bien, el empleo de unos de estos pequeños ordenadores permite definir los caracteres gráficos que el usuario necesite, de forma que no sólo podemos disponer de signos de transcripción, sino que es posible conseguir que en la pantalla - y por tanto en la impresora - aparezca un texto escrito en grafía árabe - o en cualquier otro alfabeto no latino-(4).

Estas aplicaciones del ordenador personal a la investigación en Humanidades son, ta vez, las que más llaman la atención y las que pueden liberar al investigador de una serie de trabajos tediosos y rutinarios. Sin embargo no son las únicas, y ejemplo de ello es el programa que presento a continuación.

\section{Conversión automática de fechas musulmanas a cristianas}

El programa que hemos denominado "Conversor" permite obtener la equivalencia teórica de una fecha de la hégira en el calendario cristiano. El proceso inverso no se incluye por estimar que tiene mucha menos utílidad para el investigador. Para su cálculo siempre se podrá recurrir a las Tablas de Ocaña (5) o a las de Ubieto (6) Las primeras, a partir de la edición de 1981, introducen unas regletas que, con respecto a anteriores ediciones, simplifican enormemente los cálculos. Las segundas, tras una breve introducción, reproducen todas las equivalencias día por día entre los dos calendarios, desde el año 1 al 1500 de la hégira/622 a 2077 de nuestro cómputo, interminable lista obtenida por medios informáticos.

Este programa no puede suplir a las dos obras mencionadas y a las otras muchas existentes. A no ser que se mantenga el ordenador continuamente en funcionamiento, es más cómodo y rápido, para hallar la equivalencia de un número reducido de fechas, consultar dichas obras. Pero cuando nos encontramos ante un contingente importante de fechas a convertir, el ahorro en tiempo y la mejora en seguridad que proporciona este programa es innegable. La traducción de una crónica histórica o un estudio basado en datos biográficos pueden fácilmente implicar la conversión de varios cientos de fechas, labor que, gracias al "Conversor" puede ser realizada en muy poco tiempo.

\section{CARACTERISTICAS DEL PROGRAMA}

\section{Finalidad}

Convertir automáticamente una fecha dada de la hégira en la correspondiente de la era cristiana.

(4) Aparte de los tratamientos de texto árabes lanzados por las grandes empresas de informática con destino a países árabes y de difícil - por no decir imposible - acceso en España, por el reducido número de personas que estarlan interesadas en ello y por el elevado coste de todo el software elaborado con fines comerciales, existe otro programa de tratamiento de textos realizado por $\mathrm{L}$. Molina y por mí, aún sin publicar, ya que fue pensado más como prueba de las posibilidades de los microordenadores que como programa efectivamente utilizable. Digo esto porque la reducida capacidad de memoria del ordenador utilizado (Spectrum 48K) y su limitada definiçión gráfica sólo permiten tratar textos de corta extensión $\checkmark$ con unos caracteres, que aunque perfectamente legibles, no poseen la calidad suficiente como para ser editados en reproducción fotomecánica. En un futuro próximo esperamos adaptar este programa a un microordenador de más potencia. No es preciso insistir en que la impresora a utilizar en este caso debe ser matricial.

En el artículo de Juan Luis Marcó Sánchez y Honorino Mielgo Álvarez, "Un sistema de escritura automátiça en alfạbeto áraben (Awraq, $V-V l, 1982-83,247-253$ ), puede hallarse otro intento de aproximación a esta cuestión con métodos $\checkmark$ resultados muy distintos, según parece dessprenderse de las no muy claras explicaciones de los autores.

(5) Ocaña Jiménez, Manuel, Nuevas tablas de conversión de datas islámicas a cristianas y viceversa, Màdrid, Instituto Hispa no-Árabe de Cultura-Ministerio de Cultura, 1981.

16r Ubieto Artur, Antonio-Paulo, Tablas teoricas de equivalencia diaria entre los calendarios islámico y cristiano, Zaragoza, $1984,2 v$. 


\section{Lenguaje}

BASIC.

Ha sido escrito en este lenguaje a fin de que pueda ser adaptado con muy pocas modificaciones a cualquier tipo de ordenador. Escrito en código máquina ganaría en velocidad, pero sólo podría ser utilizado en el ordenador para el que se hubiera hecho.

\section{Método del cálculo}

Basado en la cronología adoptada en las Tablas de Ocaña, el programa tiene en cuenta el desfase producido en el paso del calendario gregoriano al juliano. El método empleado consiste en hallar el número de días transcurridos desde el inicio de la hégira hasta la fecha dada. Una vez obtenida esa cifra, se calculan los años meses y días del cómputo cristiano a los que equivale $y$ se suman al 16 de julio del 622, fecha en que se dice que tuvo lugar la hégira.

Se consigue así una equivalencia teórica que necesitará ciertos ajustes, debidos a razones que ya han sido suficientemente explicadas por Ocaña en sus Tablas (7).

\section{Presentación en pantalla}

Para los nombres de los meses musulmanes se ha adoptado el sistema de transcripción empleado en mi obra «La sociedad hispanomusulmana al final del califato. Aproximación a un estudio demográfico» (8). Aunque como decía antes es posible representar en pantalla los signos habituales de transcripción, creo que ese sistema debe ser usado sólo para la impresión de textos y no en programas escritos en BASIC, que deben emplear siempre los códigos ASCII, comunes a la mayoría de los ordenadores personales.

(7) V. p. 9,33 y $130-132$

(8) Madrid: CSIC, 1985. 
10 GLSOER 5: PAPER 5: PRINT AT

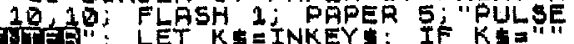
FHTER": LET KO

IS CLS PRINT THB 11; INK 3 PN $^{\prime}$ CONUERSOR". "TAE a; "O LUTS MOLINA

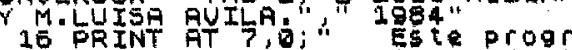
ana perinite haliarla fecha de a ara cistiana quese correspond e con una de la he-gira cegida por el usuario... " para contrin zar pulse cualquiertecla

17 LET $k$ INKEY : IF $k \$$ IN" THE N $20=017$

30 ELS : PRINT RT 4, 0; "ESGriba

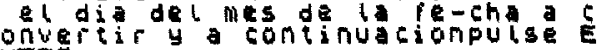
NTER,

i viene indicado con una fi el o e tipo ingiciastnocties pop pas a i t escriba -5 .

3́ IN INPUT di

37 ELS: PRINT "Introduzea el

numeródE min

mutariall =1 ravab 17

Safar =a faban =8

$r a b I * I=3$ raliadAn $=8$

rabI* II $\equiv 4$ \$aWAL $=10$

YUMAdE I =5 \&U ? Qa*da

YUMAdE II

औU J HiYYa 12

40 INPUT Mes

50 ELS NT TAB 6; "Introduzca el año": IN PUT ano

55 If dia*0 OR $d i a>30$ QR ano<1 OR mes $>13$ OR dia $\{-15$ DR Hes 11 T HEN CLS : PRINT "DATOS erTOMEOS

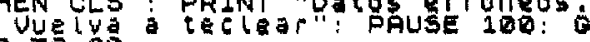
0 To 30

SO IF dia 230 AND "Datos fronés agdias solamente": "PAUSE 100: Go TQ 30

60 LET $t=0$

90 IF $d i>0$ THEN 60 TO 130

80 LET n $\Rightarrow$ ano-(INT ( $($ ano-1)/30)

* 301: 00 SUR 4000

9日 LET $E=29$

100 IF INT (mes/2) () Hes/Q THEN

LET $P=1$

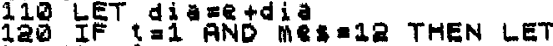

diJ日D RESTORE AQQ1: FOR $M=1$ TO ME $5 * 2$

140 READ $6 \$$

150 NEXT $n$

160 CLS : PRINT AT 6, $6 j "$ " aj" de "icsi" de "jano" "corresp onde al.

200 LET $c=I N T(\operatorname{ano-1)/30)}$

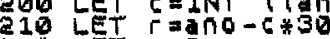

3 는다 $s=0$

30 IF $r$ S THEN GO TO 400

240 FOR $n=1$ TO $r-1$

250 LET $5=5+354$

300 GO \$UB 4000

270 NEXT $n$

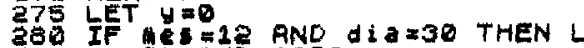
$E T H=1:$ : $n O 500$ 4000
290 IF $13=30$ AND $D=5=12$ AND 4 a THEN ELS PRINT "da to erróné

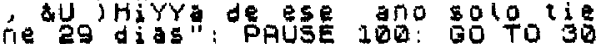
295 If $d a=30$ AND $0 E s=13$ AND $y=$ 1 THEN LET $s=5-1$

$3 O Q=E T=S=S-1$

310 LET MES $=1$. THEN 00 TO 490

350 FOR $n=1$ TO MEs-1

330 LET in $=\pi+20$

340 IF INT $(n / 2)<3 n / 2$ THEN LET

$\mathrm{m}=\mathrm{r}+\mathrm{i}$

350 NEXT $T$

400 LET $=c * 10032+s$

410 LET to talodiatm+a

420 LET ds =total - (INT $(5061 / 7)$

*71

430 IF $d S=0$ THEN LET $d s=7$

440 RESTORE 2OOL: FOR $\mathrm{n}=1$ TO 24

$+d s$

450 READ

450 NEXT $n$ t 4350721 THEN LET

talo If $t 0$ ta

500 LET $j=t o t a l+561$

510 LET $h=I N T$ (j/3461) *1461

512 LET $u=I N T(j / 1467)-260$

513 LET $a=I N T\{(v / 25\}+1$

515 IF $4>3$ THEN LET $q$ a-INT $19 /$ 4

17 IF $h>393000$ THEN LET $h=h-9$

530 LET $i=j-h$ (i / 555 )

540 LET $f=i-9 * 355$

542 IF $h>395009$ THEN LET $h=h+9$

545 LET a 6 \%8 $21+(h / 1481) * 4+9$

540 IF aC>I700 GND C $1400 \times$ INT

a 64000 . THEN 90 TO 550

547 IF a $>=1700$ AND $\mathrm{C} / 200=$ INT

(3C)(200) THEN QO TO 570

550 IF $i=1155$ THEN LET $d C=29: L$ हT b\$a"iebrero": Go To 685

500 IF $i$ ? 155 THEN LET $F=f-1$

570 RESTORE 200D: FOR $n=1$ TO 12

50 READ

580 IF $>b$ THEN LET Pan: RESTOR 2000: 60 TO 510

SOQ NEXT RÉ (Q⿱日一) -1$)$

850 REAO bE

QEU NEXT N: RESTORE QOQ1

B8Q LET $d c \equiv i-b$

1000

NK $2 ;$

1010 PRINT AT 17,0;"si desea IAP Cimifle recha obte-nida. pulse

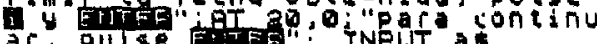
Fr PULSE E. EF"' INPUT

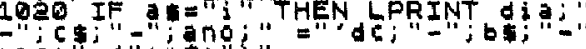
a

2000 OATA $334,304,273,243,212,18$ $1,151,120,90,59,31,0$

2001 DATA "diciembre" "muHarran" "nouiembre" "safar" "octubre" rabI* I" "septichbre" "robI* II" " "agosto" "YuRRde I" "julio", "Yu

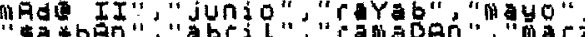

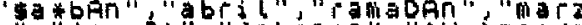

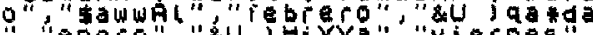

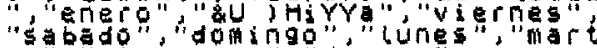
es" "ipiefcoles" "júeves" 4600 LET $y=0$ If $\pi=2$ OR $n=5$ OR $\Rightarrow 7$ OR $n=10$ OR $n=13$ OR $n=13$ oR $n=$ 16 QR $n=21$ oR $n=34$ OR $m=35$ oR $n=$ 29 THEN LET $\$=\$+1:$ LET $t=1$ : LET.

$y=2$
4001 RETURN 


\section{CONUERSOR}

(5) LUIS MOLINA Y M.LUISA RUILA.

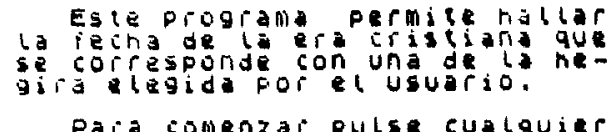
$\operatorname{tecta}$

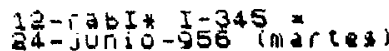

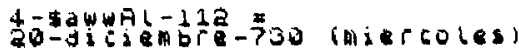

$4-53 * \tan -763$.

co-nivyo-1362 (domingo)

$7-5 a i-852=$

iviernes

Escitiba el dja del mes de le por ina a convertir y continuación PUis\& ENTER.

Si l dia viene indicado con une firase del tipo ra ga distnoches por pasars, asciba -E.

Intraduzca el numaro de mes

\begin{tabular}{|c|c|c|c|}
\hline mUHAT H hi & \pm 1 & pyeb & $\because 7$ \\
\hline saitar & .3 & co *ban & -8 \\
\hline $\cos I * I$ & .3 & ramoDAn & $=9$ \\
\hline rabI*II & -4 & EWWAL & \\
\hline YUIAAd I & -8 & ou $) 9$. & \\
\hline YUIAAdO I & $I=6$ & U IMI & \\
\hline
\end{tabular}

Introduzca el año 